\title{
Bioconversion of AS1387392: screening and characterization of actinomycetes that convert AS1387392 to AS1429716
}

\begin{abstract}
Satoshi Sasamura ${ }^{1}$, Hideyuki Muramatsu ${ }^{2}$, Shigehiro Takase ${ }^{3}$, Akihiko Fujie ${ }^{4}$, Takashi Fujii ${ }^{5,7}$, Motohiro Hino ${ }^{6}$, Kazutoshi Sakamoto ${ }^{2}$ and Michizane Hashimoto ${ }^{2}$

AS1387392 was a novel and powerful histone deacetylase inhibitor with an excellent oral absorption profile, but this compound was lacking in active moieties, which are essential to synthesize more derivatives. In our screening program to identify actinomycetes capable of converting AS1387392 to AS1429716, which has an active moiety to synthesize more derivatives, we identified 12 strains capable of efficient hydroxylation. Results of phylogenetic analysis of $16 \mathrm{~S}$ rDNA sequences suggested that these strains belonged to the genera Lentzea, Saccharopolyspora, Sphaerisporangium and Amycolatopsis. Morphological and chemical characteristics as well as results of phylogenetic analysis suggested that strain No. 7980 was a new species belonging to the genus Amycolatopsis, according to the FASTA search result of 16S rDNA gene sequence. Using these strains, we can easily produce AS1429716 as a chemical template for further chemical modifications, which may provide more effective and safer immunosuppressant.
\end{abstract}

The Journal of Antibiotics (2010) 63, 637-642; doi:10.1038/ja.2010.89; published online 28 July 2010

Keywords: Acremonium; AS1387392; AS1429716; bioconversion; histone deacetylase inhibitor; immunosuppressant

\section{INTRODUCTION}

The immunosuppressants tacrolimus and cyclosporin A dramatically changed the field of organ transplants. However, problems such as chronic rejection and other side effects based on the mode of action of these calcineurin inhibitors remain unresolved. ${ }^{1,2}$ To search for more effective, safer therapeutic agents, we continue to explore other novel immunosuppressants.

Through our efforts to address these problems, we previously identified the novel compound AS1387392, (Figure 1) a cyclic tetrapeptide produced by the fungi Acremonium sp. No. 27082. ${ }^{3}$ This compound exhibited a strong inhibitory effect against histone deacetylase and splenocyte proliferation. Further, AS1387392 showed good oral absorption, with a plasma concentration higher than that of FR235222, (Figure 1) an analog of AS1387392.4-6

As it turned out that AS1387392 would be a good chemical template, the derivatization of this compound was started. AS1387392, however, is lacking in active moieties, which are essential to synthesize more derivatives. After some feasibility studies, AS1429716 (Figure 1) with a good chemical moiety was synthesized and the activity of this compound showed as potent as that of AS1387392 in immunosuppressive activity using splenocyte proliferation assay. Although AS1429716 was obtained as an attractive template, the yield of the product by chemical synthesis method was very low. To overcome this problem, we started to study the bioconversion method to improve it.

Microorganisms are well known to be capable of hydroxylating a range of compounds. ${ }^{7-11}$ Bioconversion is practical for position- and stereo-selective hydroxylation and is a powerful tool for contributing to chemical template and metabolite production. We therefore screened actinomycetes capable of converting AS1387392 to AS1429716.

Here, we describe the screening and phylogenetic analysis for actinomycete strains, which convert AS1387392 to AS1429716, the taxonomy of strain No. 7980, and the isolation and physicochemical properties of AS1429716 in this paper.

\section{MATERIALS AND METHODS}

Microorganisms used for bioconversion

In this study, we examined $\sim 8000$ actinomycete strains isolated from soil in Japan and about 300 type strains purchased from a recognized culture collection such as American Type Culture Collection (ATCC) and Japan Collection of Microorganisms (JCM).

${ }^{1}$ Pharmacology Research Laboratories, Astellas Pharma, Ibaraki, Japan; ${ }^{2}$ Fermentation Research Division, Astellas Research Technologies, Ibaraki, Japan; ${ }^{3}$ Analysis and Pharmacokinetics Research Laboratories, Astellas Pharma, Ibaraki, Japan; ${ }^{4}$ Research Planning and Administration, Astellas Pharma, Ibaraki, Japan; ${ }^{5}$ Exploratory Research Laboratories, Astellas Pharma, Ibaraki, Japan; and ${ }^{6}$ Fermentation and Biotechnology Laboratories, Astellas Pharma, Aichi, Japan

${ }^{7}$ Current address: Kikuchi Research Center, The Chemo-Sero-Therapeutic Research Institute, 1314-1 Kyokushi Kawabe, Kikuchj-shi, Kumamoto, Japan

Correspondence: Dr S Sasamura, Pharmacology Research Laboratories, Astellas Pharma, 21 Miyukigaoka, Tsukuba-shi, Ibaraki 305-8585, Japan.

E-mail: satoshi.sasamura@jp.astellas.com

Received 9 February 2010; revised 13 June 2010; accepted 16 June 2010; published online 28 July 2010 


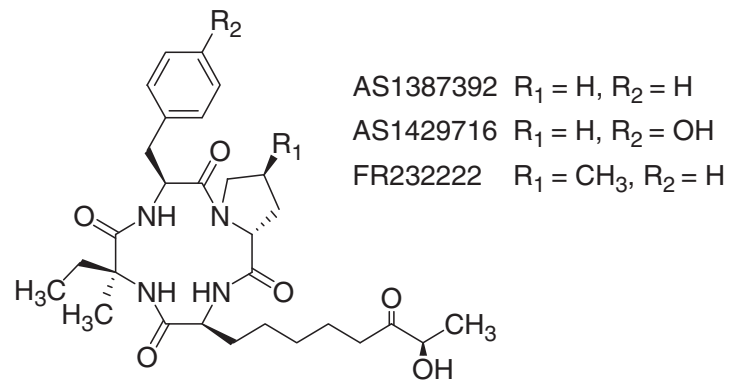

Figure 1 Structures of AS1387392, AS1429716 and FR235222.

\section{Culture media}

Medium A consisted of cornmeal 1\%, MS\#3600 6\% (Nihon Shokuhin Kako, Tokyo, Japan), Pharmamedia $1.2 \%$ (Traders Protein, Lubbock, USA), dried yeast $0.8 \%, \mathrm{KH}_{2} \mathrm{PO}_{4} 0.3 \%, \mathrm{MgSO}_{4} \cdot 7 \mathrm{H}_{2} \mathrm{O} 0.3 \%$ and $\mathrm{FeSO}_{4} \cdot 7 \mathrm{H}_{2} \mathrm{O} 0.02 \%$, $\mathrm{pH}$ 6.5. Medium B consisted of glucose $2 \%$, MS\#3600 $5 \%$, potato protein $1.5 \%$, wheat germ $1.5 \%$ and $\mathrm{CaCO}_{3} 0.2 \%, \mathrm{pH}$ 6.5. Medium $\mathrm{C}$ consisted of glucose $0.5 \%$, MS\#3600 $2.5 \%$, dried yeast $0.5 \%$, soybean flour $1 \%$, L-asparagine $0.2 \%$, $\mathrm{CuSO}_{4} \cdot 5 \mathrm{H}_{2} \mathrm{O} 0.0005 \%, \mathrm{FeSO}_{4} \cdot 7 \mathrm{H}_{2} \mathrm{O} 0.001 \%, \mathrm{MgSO}_{4} \cdot 7 \mathrm{H}_{2} \mathrm{O} 0.05 \%$ and $\mathrm{CaCO}_{3}$ $0.2 \%, \mathrm{pH}$ 6.5. Medium D consisted of glucose $0.5 \%$, sucrose $0.5 \%$, oatmeal $0.5 \%$, yeast extract $0.2 \%$, peptone $0.5 \%$, peanut powder $0.5 \%$, humic acid $0.01 \%$, Tween $800.1 \%$ and $\mathrm{CaCO}_{3} 0.2 \%$, pH 7.0. Medium E consisted of glycerol $6 \%$, soybean flour $1 \%$ and corn steep liquor 3\% (Nihon Shokuhin Kako, Tokyo, Japan), pH 6.5.

\section{Culture and bioconversion conditions for screening}

Actinomycete strains were grown in tubes $(17 \mathrm{~mm} \times 100 \mathrm{~mm})$ containing $1.5 \mathrm{ml}$ of media A, B or C. The culture tubes were inoculated with a loopful of the actinomycete plate culture, and then incubated on a shaker at 250 r.p.m. at $30^{\circ} \mathrm{C}$ for 4 or 6 days, depending on cell growth. After incubation, $0.35 \mathrm{ml}$ of culture broth was transferred to another tube containing $170 \mathrm{~mm}$ phosphate buffer (pH 6.5) and $1.7 \mathrm{mg} \mathrm{ml}^{-1}$ AS1387392 in $0.15 \mathrm{ml}$ of deionized water.

\section{Sample analysis for screening}

The reaction mixtures were combined with $0.5 \mathrm{ml}$ of acetone and centrifuged (3000 r.p.m., $10 \mathrm{~min}$ ), and then the supernatant was transferred to HPLC vials. Primary analysis was performed using our HPLC system (L-4000 UV detector, L-6000 pump and L-7300 column oven; Hitachi High-Technologies, Tokyo, Japan) using an ODS column (Mightysil RP-18 GP $250 \mathrm{~mm} \times 4.6 \mathrm{~mm}$ ID; Kanto chemical, Tokyo, Japan). The mobile phase was $45 \%$ aqueous acetonitrile and the flow rate was $1.0 \mathrm{ml} \mathrm{min}^{-1}$. The detection wavelength was set at $210 \mathrm{~nm}$ and the column was heated at $50{ }^{\circ} \mathrm{C}$. Under these conditions, AS1429716 and AS1387392 were eluted at a retention time of 4.6 and $9.8 \mathrm{~min}$. Secondary analysis was performed by liquid chromatography-mass spectrometry (YMC ODS-AM AM-3C1-3 $100 \mathrm{~mm} \times 2.0 \mathrm{~mm}$ ID, $3 \mu \mathrm{m}$ (YMC, Kyoto, Japan; flow rate: $0.2 \mathrm{ml} \mathrm{min}^{-1}$, mobile phase: $30 \%$ aqueous acetonitrile, detection: MS and UV absorption at $210 \mathrm{~nm}$, column temperature: $50^{\circ} \mathrm{C}$ ) using an Agilent HP1100 (Hewlett-Packard, Palo Alto, CA, USA). Under these conditions, AS1429716 was eluted at a retention time of $8.1 \mathrm{~min}$ and showed a molecular ion peak at $m / z 559[\mathrm{M}+\mathrm{H}]^{+}$.

\section{$16 S$ rDNA sequencing and phylogenetic analysis}

The 16S rDNA sequence was determined using the method developed by Nakagawa et al. ${ }^{12} 16 \mathrm{~S}$ rDNA sequences of type strains were obtained from the DNA Data Bank of Japan database, (http://www.ddbj.nig.ac.jp) and a phylogenetic tree was constructed using the alignment program ClustalW. ${ }^{13}$

\section{Taxonomic characteristics of strain No. 7980}

Strain No. 7980 was isolated from a soil sample collected in Nagano Prefecture, Japan. We conducted our taxonomic study of strain No. 7980 using the methods and media described by Shirling and Gottlieb, ${ }^{14}$ and Waksman. ${ }^{15}$
All observations were made after 14 days' cultivation at $30^{\circ} \mathrm{C}$. Morphological observations were made using an optical microscope and a scanning electron microscope on cultures grown on yeast extract-starch agar containing $0.08 \mathrm{~g}$ of yeast extract (Daigo Eiyo, Osaka, Japan), $0.4 \mathrm{~g}$ of soluble starch and $16 \mathrm{~g}$ of agar in 11 of tap water (pH adjusted to 7.2 with $1 \mathrm{~N} \mathrm{NaOH}$ before sterilization). The range of temperature for growth was determined on yeast extract-starch agar containing $2 \mathrm{~g}$ of yeast extract, $10 \mathrm{~g}$ of soluble starch and $16 \mathrm{~g}$ of agar in 11 of tap water ( $\mathrm{pH}$ adjusted to 7.2 with $1 \mathrm{~N} \mathrm{NaOH}$ before sterilization). Carbon utilization was determined on Pridham and Gottlieb's medium. ${ }^{16}$ All color names used in this study were taken from Methuen Handbook of Colour. ${ }^{17}$ Cell preparation and diaminopimelic acid isomer detection were conducted following the procedure developed by Becker et al. ${ }^{18}$

\section{Culture and bioconversion using strain No. 7980}

Aqueous seed medium D $(30 \mathrm{ml})$ was poured into a 100-ml Erlenmeyer flasks and sterilized at $121^{\circ} \mathrm{C}$ for $30 \mathrm{~min}$. The seed flasks were then inoculated with a loopful of the strain No. 7980 plate culture and shaken on a shaker at $30^{\circ} \mathrm{C}$ for three days. After incubation, $0.6 \mathrm{ml}$ of the seed culture was then transferred to 100 -ml Erlenmeyer flasks containing $30 \mathrm{ml}$ of medium E, and these flasks were shaken at $30^{\circ} \mathrm{C}$ for two days. After this cultivation, $35 \mathrm{ml}$ of the culture broth and $15 \mathrm{ml}$ of solution containing $170 \mathrm{~mm}$ phosphate buffer $(\mathrm{pH} 6.5)$ and $1.3 \mathrm{mg} \mathrm{ml}^{-1}$ AS1387392 were transferred to $500-\mathrm{ml}$ baffled flasks, and then incubated at $30^{\circ} \mathrm{C}$ for $5 \mathrm{~h}$. Bioconversion stage contained $50 \mathrm{ml}$ of reaction mixtures in a $500-\mathrm{ml}$ baffled flask.

\section{RESULTS}

Screening of microorganisms for ability to convert AS1387392 to AS1429716

Preliminary screening was carried out for $\sim 300$ strains of fungi and actinomycetes isolated from soil and leaf. Of these, only five strains of actinomycetes were able to convert AS1387392 to AS1429716. Given these findings, we decided to conduct subsequent screening using only actinomycete strains.

To assess bioconversion activity, we conducted HPLC analysis on $\sim 8000$ strains isolated from soil in Japan and about 300 type strains of various actinomycete species. After secondary analysis using liquid chromatography-mass spectrometry, we analyzed phylogenetically 12 strains, including 3 type strains, capable of converting AS1387392 to AS1429716.

\section{Phylogenetic analysis of bioconversion strains}

Phylogenetic analysis based on $16 \mathrm{~S}$ rDNA sequences showed that the strains capable of bioconversion belonged to the genera Lentzea, Saccharopolyspora, Sphaerisporangium and Amycolatopsis. A phylogenetic tree is shown in Figure 2, and a complete list of the strains capable of bioconversion is shown in Table 1. Relevant strains included high converting yield strains, such as Lentzea sp. No. 86, Sphaerisporangium sp. No. 2768, Amycolatopsis azurea JCM 3275 and Amycolatopsis sp. No. 7980. No Streptomyces were found capable of converting AS1387392 to AS1429716.

\section{Taxonomic characteristics of strain No. 7980}

The substrate mycelium developed well and branched irregularly, and hyphal segment formation was observed. This strain produced few aerial mycelia, and no spores were observed. Sclerotic granules, sporangia and motile spores or fragments were also not observed.

Cultural and physiological characteristics are described in Tables 2 and 3, respectively. Aerial mycelia were rarely observed. Reverse side growth color ranged from yellowish-white to orange. No melanoid pigments were produced in the tryptone-yeast extract broth or peptone-yeast extract-iron agar (ISP-6). Soluble pigments were produced in the inorganic salts-starch agar (ISP-4). Mycelial mass color 


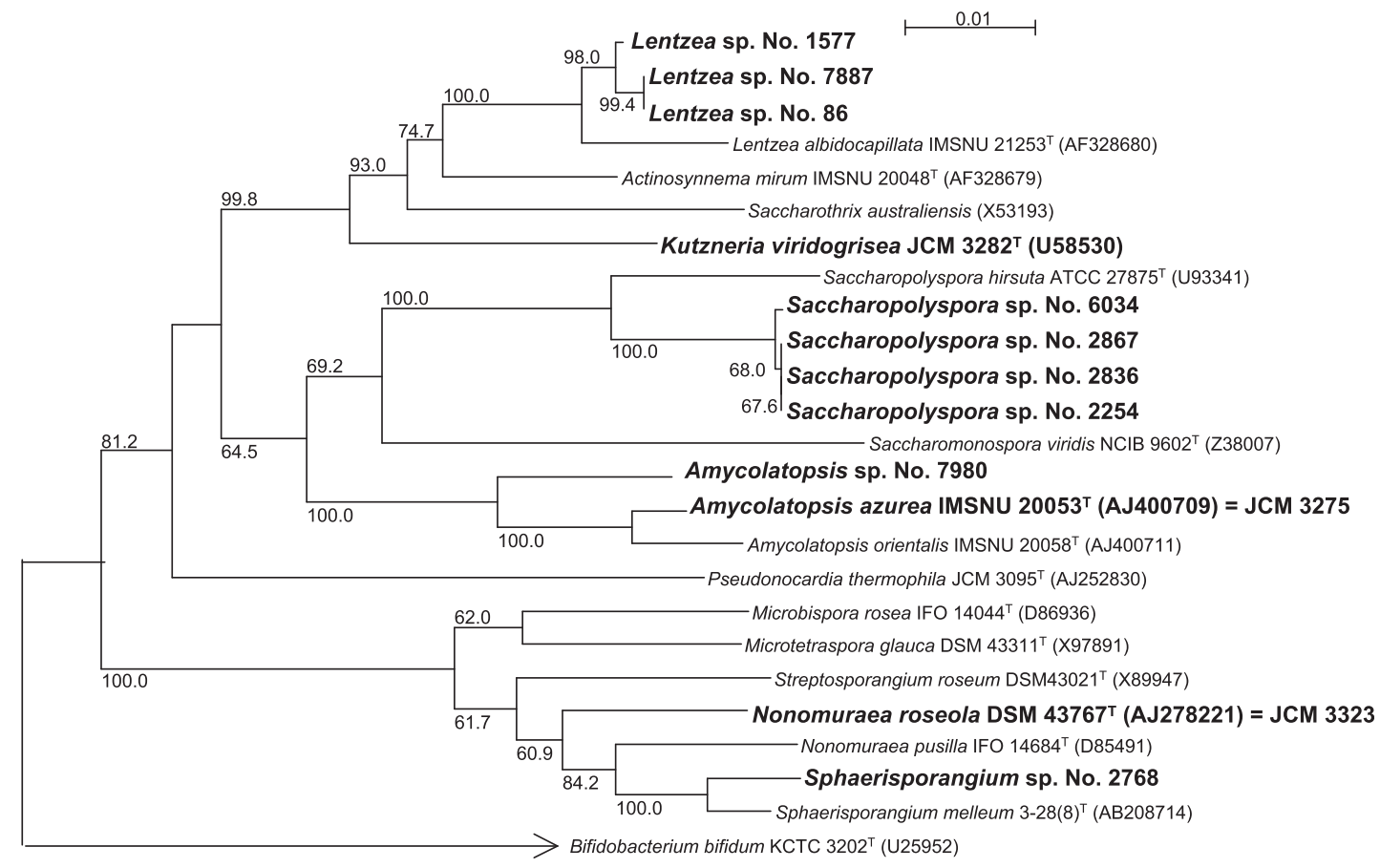

Figure 2 Phylogenetic tree showing actinomycete strains capable of converting AS1387392 to AS1429716 based on 16S rDNA sequence (almost fulllength ca 1400 nucleotides). The numbers on the branches are confidence limits (expressed as percentages) estimated from a bootstrap analysis with 1000 replicates (above $50 \%$ are indicated). Bar, 0.01 substitutions per nucleotide position.

Table 1 Actinomycetes strains capable of converting AS1387392 to AS1429716

\begin{tabular}{lc}
\hline Microorganism & Bioconversion ratio \\
\hline Lentzea sp. No. 86 & ++ \\
Lentzea sp. No. 1577 & + \\
Lentzea sp. No. 7887 & + \\
Amycolatopsis azurea JCM 3275 & ++ \\
Amycolatopsis sp. No. 7980 & ++ \\
Saccharopolyspora sp. No. 2254 & + \\
Saccharopolyspora sp. No. 2836 & + \\
Saccharopolyspora sp. No. 2867 & + \\
Saccharopolyspora sp. No. 6034 & + \\
Kutzneria viridogrisea JCM 3282 & + \\
Nonomuraea roseola JCM 3323 & + \\
Sphaerisporangium sp. No. 2768 & ++ \\
\hline a $0.5-15 \%$.+ $>15 \%$ Bioconversion ratio was expressed as the value of the AS1429716
\end{tabular}
per initial AS1387392.

Table 2 Cultural characteristics of strain No. 7980

\begin{tabular}{lllll}
\hline Medium & Growth & Aerial mycelia & Color of reverse surface & Soluble pigment \\
\hline ISP-2 & Weak & Not observed & Orange (5B8) & Not detected \\
ISP-3 & Weak & Not observed & Yellowish-white (3A2) & Not detected \\
ISP-4 & Weak & Not observed & Yellowish-white (4A2) & Brown (6D7) \\
ISP-5 & Weak & Not observed & Light orange (5A4) & Not detected \\
ISP-6 & Weak & Not observed & Orange (5A6) & Not detected \\
ISP-7 & Weak & Not observed & Light yellow (4A5) & Not detected \\
\hline
\end{tabular}

Abbreviations: ISP-2, yeast-malt extract agar; ISP-3, oatmeal agar; ISP-4, inorganic salts-starch agar; ISP-5, glycerol-asparagine agar; ISP-6, peptone-yeast extract-iron agar; ISP-7, tyrosine agar
Table 3 Physiological characteristics of strain No. 7980

\begin{tabular}{lc}
\hline Characteristic & Value \\
\hline Temperature range for growth $\left({ }^{\circ} \mathrm{C}\right)$ & $8-34$ \\
Optimum temperature for growth $\left({ }^{\circ} \mathrm{C}\right)$ & 32 \\
Melanoid pigments produced & - \\
Soluble pigments produced & + \\
& \\
Carbon utilization & + \\
D-glucose & - \\
Sucrose & \pm \\
$D$-Xylose & + \\
D-fructose & - \\
L-rhamnose & + \\
Raffinose & + \\
L-arabinose & + \\
Inositol & + \\
D-mannnitol & + \\
\hline,+ utilized;,- not utilized; \pm weakly utilized & + \\
\end{tabular}

was not $\mathrm{pH}$ sensitive. Meso-diaminopimelic acid was detected in whole-cell hydrolysates of this strain. Similarity values of $16 \mathrm{~S}$ rDNA between strain No. 7980 and members of the genus Amycolatopsis were 93.2-96.1\%, and both formed a single cluster on the phylogenetic tree (Figure 3).

On the basis of the morphological and chemical characteristics and phylogenetic analysis described above, we concluded that strain No. 7980 belonged to the genus Amycolatopsis. ${ }^{19,20}$ Therefore, this strain was designated as Amycolatopsis sp. No. 7980. 


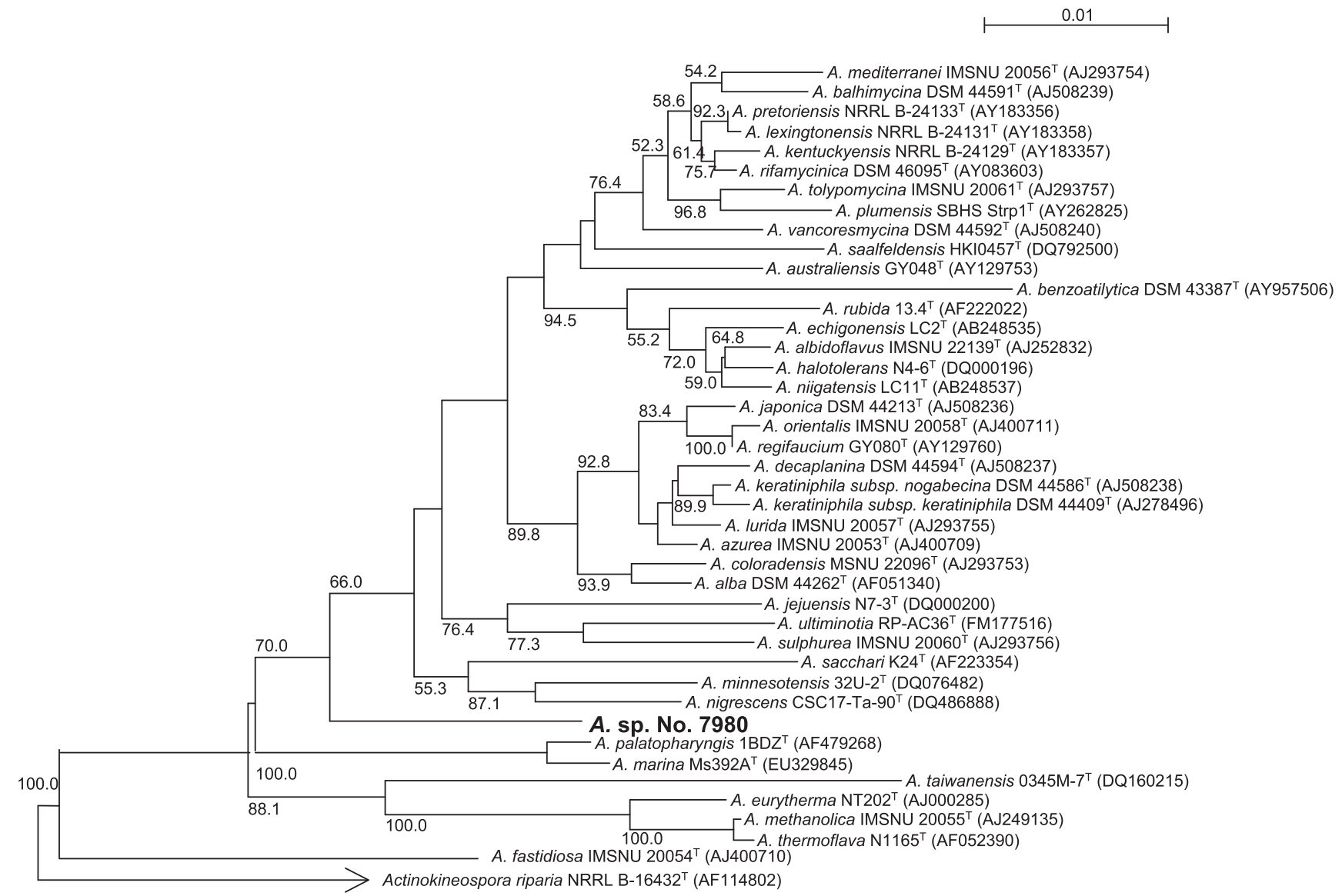

Figure 3 Phylogenetic tree showing the position of strain No. 7980 based on 16S rDNA sequence (almost full-length ca 1400 nucleotides). The numbers on the branches are confidence limits (expressed as percentages) estimated from a bootstrap analysis with 1000 replicates (above 50\% are indicated). Bar, 0.01 substitutions per nucleotide position.

\section{Isolation and physicochemical properties of AS1429716}

After reaction, the $200 \mathrm{ml}$ mixtures obtained from four baffled flasks were extracted with $200 \mathrm{ml}$ of methanol. The methanol extract filtrate was concentrated to an aqueous solution and extracted with ethyl acetate. The organic layer was concentrated and applied to a column of $20 \mathrm{ml}$ Daiso ODS-B (Daiso, Osaka, Japan). The column was eluted with $30 \%$ aqueous acetonitrile. Further purification was accomplished by preparative HPLC (Mightysil RP-18 GP $250 \mathrm{~mm} \times 4.6 \mathrm{~mm}$ ID; Kanto Chemical; flow rate: $1 \mathrm{ml} \mathrm{min}^{-1}$, mobile phase: $25 \%$ aqueous acetonitrile, temperature: $50^{\circ} \mathrm{C}$, detection: UV absorption at $210 \mathrm{~nm}$ ). The active fraction was concentrated in vacuo and extracted using ethyl acetate, and the solvent layer was dried up and dissolved in tert-butyl alcohol. Subsequent lyophilization produced $4.7 \mathrm{mg}$ of AS1429716 as a white powder.

Physicochemical properties of AS1429716 are described in Table 4. The compound was soluble in methanol, ethyl acetate and dimethyl sulfoxide, and slightly soluble in water. Positive-color reactions were observed with iodine vapor, $\mathrm{FeCl}_{3}$ and $\mathrm{Ce}\left(\mathrm{SO}_{4}\right)_{2}$ $\mathrm{H}_{2} \mathrm{SO}_{4}$, and negative reactions during Molisch's, Ehrlich's and Ninhydrin reactions. The observed specific rotation was $-75^{\circ}$ (c 0.2, methanol), and the exact molecular mass (as determined by ESI-TOF-MS) was $559.3122 \mathrm{Da}\left([\mathrm{M}+\mathrm{H}]^{+}\right)$, corresponding to the molecular formula $\mathrm{C}_{29} \mathrm{H}_{42} \mathrm{~N}_{4} \mathrm{O}_{7}$ (theoretical $[\mathrm{M}+\mathrm{H}]^{+}=$ $559.3132 \mathrm{Da})$.
Table 4 Physicochemical properties of AS1429716

\begin{tabular}{|c|c|}
\hline Characteristic & Value \\
\hline Appearance & White powder \\
\hline$[\alpha]_{D}^{23}$ & $-75^{\circ}(c 0.2, \mathrm{MeOH})$ \\
\hline \multicolumn{2}{|l|}{ ESI-TOF-MS $(\mathrm{m} / \mathrm{z})$} \\
\hline Observed & $559.3122[\mathrm{M}+\mathrm{H}]^{+}$ \\
\hline Calculated & $559.3132[\mathrm{M}+\mathrm{H}]^{+}$ \\
\hline Molecular formula & $\mathrm{C}_{29} \mathrm{H}_{42} \mathrm{~N}_{4} \mathrm{O}_{7}$ \\
\hline UV $\lambda_{\max }(\mathrm{MeOH}) \mathrm{nm}(\log \varepsilon)$ & 223 (sh), 276 (3.75) \\
\hline \multicolumn{2}{|l|}{ Color test } \\
\hline Positive & $\mathrm{I}_{2}, \mathrm{Ce}\left(\mathrm{SO}_{4}\right)_{2}-\mathrm{H}_{2} \mathrm{SO}_{4}, \mathrm{FeCl}_{3}$ \\
\hline Negative & $\begin{array}{l}\text { Molisch's reaction, Ninhydrin reaction, } \\
\text { Ehrlich's reaction }\end{array}$ \\
\hline \multicolumn{2}{|l|}{ Solubility } \\
\hline Soluble & $\begin{array}{l}\text { Methanol, ethyl acetate, dimethyl } \\
\text { sulfoxide }\end{array}$ \\
\hline Slightly soluble & Water \\
\hline TLC (Rf value) $)^{a}$ & $0.23^{b}$ \\
\hline
\end{tabular}

aPlate: silica gel $60 \mathrm{~F}_{254}$ (E. Merck, Darmstadt, Germany), ethyl acetate ${ }^{\mathrm{b}} \mathrm{Rf}$ value of AS1387392 was 0.42 from reference 3. 
Table $5{ }^{1} \mathrm{H},{ }^{13} \mathrm{C}$ data of AS1429716 and ${ }^{13} \mathrm{C}$ data of AS1387392

\begin{tabular}{|c|c|c|c|c|}
\hline & & AS14 & & AS1387392 \\
\hline & Position & $\delta_{H^{a}}$ & $\delta_{C}{ }^{\mathrm{b}}$ & $\delta_{C}{ }^{c}$ \\
\hline Aoh & 1 & & 174.2 & 174.1 \\
\hline & 2 & 4.20 & 54.4 & 54.4 \\
\hline & 3 & $1.83,1.60$ & 28.8 & 28.7 \\
\hline & 4 & 1.30 & 25.3 & 25.3 \\
\hline & 5 & 1.33 & 28.8 & 28.8 \\
\hline & 6 & 1.62 & 23.2 & 23.2 \\
\hline & 7 & $2.50,2.44$ & 37.3 & 37.3 \\
\hline & 8 & & 212.4 & 212.4 \\
\hline & 9 & 4.23 & 72.6 & 72.6 \\
\hline & 10 & 1.39 & 19.8 & 19.8 \\
\hline & 11 & 7.10 & & \\
\hline Pro & 12 & & 171.9 & 171.8 \\
\hline & 13 & 4.68 & 57.8 & 57.8 \\
\hline & 14 & $2.35,1.76$ & 24.7 & 24.7 \\
\hline & 15 & $2.20,1.80$ & 25.0 & 25.0 \\
\hline & 16 & $3.83,3.27$ & 46.9 & 47.0 \\
\hline & 17 & & & \\
\hline & 18 & - & - & - \\
\hline Tyr & 19 & & 172.9 & 172.8 \\
\hline & 20 & 5.12 & 53.6 & 53.3 \\
\hline & 21 & $3.17,2.88$ & 34.9 & 35.8 \\
\hline & 22 & & 129.0 & 137.0 \\
\hline & 23,27 & 7.08 & 130.3 & 129.0 \\
\hline & 24,26 & 6.73 & 115.4 & 128.6 \\
\hline & 25 & & 154.4 & 126.7 \\
\hline & 28 & 7.54 & & \\
\hline Iva & 29 & & 175.7 & 175.6 \\
\hline & 30 & & 63.1 & 63.1 \\
\hline & 31 & $2.31,2.17$ & 27.9 & 27.9 \\
\hline & 32 & 0.85 & 8.4 & 8.4 \\
\hline & 33 & 1.29 & 22.4 & 22.4 \\
\hline & 34 & 5.88 & & \\
\hline
\end{tabular}

${ }^{\mathrm{a}} \delta_{\mathrm{H}} 500 \mathrm{MHz}$ in $\mathrm{CDCl}_{3}$.

${ }^{b_{\mathrm{C}}} 125 \mathrm{MHz}$ in $\mathrm{CDCl}_{3}$

${ }^{c} \delta_{\mathrm{C}}$ from reference 3 .

${ }^{1} \mathrm{H}$ and ${ }^{13} \mathrm{C}$ NMR spectra were recorded with a Bruker AVANCE 500 NMR spectrometer with tetramethylsilane used as an internal standard.

\section{Structure elucidation}

ESI-TOF-MS analysis of AS1429716 afforded a molecular formula $\mathrm{C}_{29} \mathrm{H}_{42} \mathrm{~N}_{4} \mathrm{O}_{7}$ indicating the presence of an extra oxygen atom compared with AS1387392 as previously reported. ${ }^{3}$ Judging from the ${ }^{1} \mathrm{H}$ NMR spectra, the position of the oxygen was determined to be para-position of benzene moiety. An A2B $2{ }^{1} \mathrm{H}$ signal pattern $(\delta 7.08$ $(2 \mathrm{H}, \mathrm{d}, \mathrm{J}=8 \mathrm{~Hz})$ and $6.73(2 \mathrm{H}, \mathrm{d}, \mathrm{J}=8 \mathrm{~Hz}))$ was typical of $1,4-$ disubstituted benzene unit. Replacement of phenylalanine in AS1387392 by tyrosine gave the structure of AS1429716. The structure was further supported by the fact that ${ }^{13} \mathrm{C}$ data of AS1429716 were quite similar to that of AS1387392 except for tyrosine part. Complete ${ }^{1} \mathrm{H}$ and ${ }^{13} \mathrm{C}$ NMR assignment was made by COSY, HSQC, HMBC and summarized in Table 5 together with ${ }^{13} \mathrm{C}$ data of AS1387392

\section{DISCUSSION}

In the field of immunosuppressant, AS1429716 is needed as chemical template; however, this compound is difficult to achieve by chemical reaction. Given that previous studies have found bioconversion to be extremely successful in hydroxylation of various compounds, we considered that production of AS1429716 from AS1387392 with bioconversion to help further chemical analyses. To identify microorganisms capable of converting AS1387392 to AS1429716, we screened some 8000 actinomycete strains. Of these, 12 were found capable of hydroxylation, and the activity of 7 was 'highly efficient'.

Phylogenetic analysis based on $16 \mathrm{~S}$ rDNA sequence was performed on the strains found capable of this conversion. Interestingly, all of these strains were non-Streptomyces actinomycetes, with no Streptomyces identified during screening though Streptomyces have been found to be able to hydroxylate various compounds. ${ }^{21-23}$ More than half of 300 actinomycete type strains we have screened, belong to the genus Streptomyces. On application of conventional isolation techniques, more than half of the strains from soil belonged to the genus Streptomyces. ${ }^{24}$ These previous findings therefore indicated that non-Streptomyces strains were ideal for screening for ability to convert AS1387392 to AS1429716, at least under the present culture and bioconversion conditions.

Observations of morphological and chemical characteristics and phylogenetic analysis indicated that strain No. 7980 belonged to the genus Amycolatopsis. ${ }^{19,20}$ The criterion for similarity using comparison of $16 \mathrm{~S}$ rDNA sequence is at least $97 \%$ homology may indicate that two species are different in the present species definition in bacteriology. ${ }^{25}$ No Amycolatopsis species showed more than $97 \%$ similarity value with that of strain No. 7980. We therefore postulated that strain No. 7980 represented a new species under the genus Amycolatopsis.

In this study, we successfully found bacterial strains capable of producing AS1429716, which until now had been chemically inaccessible. With these new findings, further derivation studies may yield still more effective and safer immunosuppressant.

\section{CONFLICT OF INTEREST}

The authors declare no conflict of interest.

\section{ACKNOWLEDGEMENTS}

We thank Ms Michiko Iitsuka for conducting the ESI-TOF-MS measurement of AS1429716 and Dr Yasuharu Urano for chemical synthesis of AS1429716, ${ }^{26}$ and Ms Mayumi Amako for assistance throughout this work.

1 Su, Q. L., Weber, M., Hir, M. L., Zenke, G. \& Ryffel, B. Nephrotoxicity of cyclosporin A and FK506: inhibition of calcineurin phosphatase. Ren. Physiol. Biochem. 18, 128-139 (1995).

2 Bechstein, W. O. Neurotoxicity of calcineurin inhibitors: impact and clinical management. Transpl. Int. 13, 313-326 (2000).

3 Sasamura, S. et al. AS1387392, a novel immunosuppressive cyclic tetrapeptide compound with inhibitory activity against mammalian histone deacetylase. J. Antibiot. 63: (in submitted) (2010).

4 Mori, $\mathrm{H}$. et al. FR235222, a fungal metabolite, is a novel immunosuppressant that inhibits mammalian histone deacetylase (HDAC). I. Taxonomy, fermentation, isolation and biological activities. J. Antibiot. 56, 72-79 (2003).

5 Mori, H. et al. FR235222, a fungal metabolite, is a novel immunosuppressant that inhibits mammalian histone deacetylase (HDAC). III. Structure determination. J. Antibiot. 56, 181-185 (2003).

6 Mori, H. et al. FR235222, a fungal metabolite, is a novel immunosuppressant that inhibits mammalian histone deacetylase (HDAC). II. Biological activities in animal models. J. Antibiot. 56, 80-86 (2003).

7 Serizawa, N., Serizawa, S., Furuya, K., Okazaki, T. \& Terahara, A. Microbial hydroxylation of ML-236B (compactin). Studies on microorganisms capable of 3 beta-hydroxylation of ML-236B, J. Antibiot. 36, 887-891 (1983).

8 Sasaki, J. et al. Transformation of vitamin $D_{3}$ to $1 \alpha, 25$-dihydroxyvitamine $D_{3}$ via 25-hydroxyvitamine $\mathrm{D}_{3}$ using Amycolata sp. strains. Appl. Microbiol. Biotechnol. 38, 152-157 (1992).

9 Schulman, M., Doherty, P., Zink, D. \& Arison, B. Microbial conversion of avermectins by Saccharopolyspora erthraea hydroxylation at C-27. J. Antibiot. 47, 372-375 (1994). 
10 Nakagawa, K. \& Torikata, A. Microbial conversion of milbemycins: 30-oxidation of milbemycin A4 and related compounds by Amycolatata autotrophica and amycolatopsis mediterranei. J. Antibiot. 43, 1321-1328 (1990).

11 Hall, R. M. et al. The production of novel sordarin analogues by transformation. J. Antibiot. 54, 948-957 (2001).

12 Nakagawa, Y. \& Kawasaki, H. Identification Manual of Actinomycetes. Edited by The Society for Actinomycetes Japan 83-117 (Business Center for Academic Societies Japan, Tokyo, 2001) (in Japanese).

13 Thompson, J. D., Higgins, D. G. \& Gibson, T. J. CLUSTAL W improving the sensitivity of progressive multiple sequence alignment through sequence weighting, position-specific gap penalties and weight matrix choice. Nucleic Acids Res. 22, 4673-4680 (1994).

14 Shirling, E. B. \& Gottlieb, D. Methods for characterization of Streptomyces species. Int. J. Syst. Bacteriol. 16, 313-340 (1966).

15 Waksman, S. A. The Actinomycetes Vol. 2: Classification, identification and description of genera and species (Williams and Wilkins, Baltimore, 1961).

16 Pridham, T. G. \& Gottlieb, D. The utilization of carbon compounds by some Actinomycetales as an acid for species determination. J. Bacteriol. 56, 107-114 (1948).

17 Kornerup, A. \& Wanscher, J. H Methuen Handbook of Colour (Methuen, London, 1978).

18 Becker, B., Lechevalier, M. P., Gordon, R. E. \& Lechevalier, H. A. Rapid differentiation between Nocardia and Streptomyces by paper chromatography of whole-cell hydrolysates. Appl. Microbiol. 12, 421-423 (1964).
19 Lechevalier, M. P. \& Lechevalier, H. A. Chemical composition as a criterion in the classification of aerobic actinomycetes. Int. J. Syst. Bacteriol. 20, 435-443 (1970).

20 Williams, S. T. Bergey's Manual of Systematic Bacteriology, Vol. 4. (Williams and Wilkins, Baltimore, 1989).

21 Tanaka, M. et al. Microbial hydroxylation of Zofimarin, a sordarin-related antibiotic. J. Antibiot. 55, 437-441 (2002).

22 Matsuoka, T. et al. Purification and characterization of cytochrome $P-450_{\text {sca }}$ from Streptomyces carbophilus. Eur. J. Biochem. 184, 707-713 (1989).

23 Berrie, J. R., Williams, A. D. R. \& Smith, K. E. Microbial transformations of steroid-XI. Progesterone transformation by Streptomyces roseochromogenens-purification and characterization of the 16 $\alpha$-hydroxylase system. J. Steroid Biochem. Mol. Biol. 71, 153-165 (1999).

24 Muramatsu, H., Shahab, N., Tsurumi, Y. \& Hino, M. A comparative study of malaysian and Japanease Actinomycetes using a simple identification method based on partial 16S rDNA sequence. Actinomycetologica 17, 33-43 (2003).

25 Stackebrandt, E. \& Goebel, B. M. Taxonomic note: a place for DNA-DNA reassociation and 16S rRNA sequence analysis in the present species definition in bacteriology. Int. J. Syst. Bacteriol. 44, 846-849 (1994).

26 Satoh, S. et al. Cyclic tetrapeptide compound and use thereof. WO 03/057722 July 17 (2003). 